\title{
Online therapy: an added value for inpatient routine care? Perspectives from mental health care professionals
}

\author{
Julia Sander ${ }^{1}$ (1) $\cdot$ Felix Bolinski ${ }^{2,3} \cdot$ Sandra Diekmann $^{4} \cdot$ Wolfgang Gaebel $^{4,5} \cdot$ Kristina Günther $^{1} \cdot$ Iris Hauth $^{1}$. \\ Andreas Heinz $^{1,6} \cdot$ Annet Kleiboer ${ }^{2,3} \cdot$ Heleen Riper $^{2,3} \cdot$ Nadine Trost $^{4} \cdot$ Oyono Vlijter ${ }^{7}$ Jürgen Zielasek ${ }^{4,5}$. \\ Gabriel Gerlinger ${ }^{1}$
}

Received: 25 September 2020 / Accepted: 3 March 2021 / Published online: 16 March 2021

(c) Springer-Verlag GmbH Germany, part of Springer Nature 2021

\begin{abstract}
Internet-delivered interventions can be effective in treating mental disorders. However, their rate of use in German psychiatric inpatient routine care is low. The current study aimed to investigate the attitude of mental health care professionals working in inpatient care regarding internet-delivered interventions, including presumed benefits, barriers and facilitators. In total, 176 health professionals from ten inpatient psychiatric hospitals throughout Germany were surveyed on site. The professionals' attitude towards internet-delivered interventions in inpatient care was assessed by an adapted version of the 'Attitude toward Telemedicine in Psychiatry and Psychotherapy' (ATTiP) questionnaire. To identify benefits, barriers and facilitators, we developed open-response questions that were based on the 'Unified Theory of Acceptance and Use of Technology' (UTAUT) and analyzed by a qualitative content analysis. Professionals reported little experience or knowledge about internet-delivered interventions. Their attitude towards internet-delivered interventions in psychiatric inpatient care was rather indifferent. The most frequently mentioned potential benefits were an optimised treatment structure and patient empowerment; the most frequently anticipated barriers were too severe symptoms of patients, the feared neglect of face-to-face contacts and insufficient technical equipment; and the most frequently mentioned facilitators were high usability of the internet-based intervention, a sufficient functional level of the patient and further education of staff. For successful implementation in the inpatient sector, internet-delivered interventions must be adapted to the special needs of severely mentally ill patients and to the hospital management systems and workflow. In addition, technical preconditions (internet access, devices) must be met. Last, further education of mental health care professionals is needed.
\end{abstract}

Keywords E-mental health · Professionals' attitude $\cdot$ Psychiatric inpatient care $\cdot$ Barriers $\cdot$ Benefits $\cdot$ Facilitators to implementation

Julia Sander

j.sander@dgppn.de

1 German Association for Psychiatry, Psychotherapy and Psychosomatics E.V. (DGPPN), Reinhardtstraße 27b, 10117 Berlin, Germany

2 Vrije Universiteit Amsterdam, Faculty of Behavioural and Movement Sciences, Clinical, Neuro- and Developmental Psychology, Amsterdam, The Netherlands

3 Amsterdam Public Health Research Institute, Faculty of Behavioural and Movement Sciences, Vrije Universiteit Amsterdam, Amsterdam, The Netherlands
4 LVR-Institute for Healthcare Research, Cologne, Germany

5 Medical Faculty, Heinrich-Heine-University, Düsseldorf, Germany

6 Department of Psychiatry and Psychotherapy, Charité, Universitätsmedizin Berlin, Campus Charité Mitte, Berlin, Germany

7 ARQ National Psychotrauma Centre, Diemen, The Netherlands 


\section{Introduction}

There is growing evidence for the effectiveness of e-mental health interventions [1-4]. In this study, we use the terms e-mental health and online therapy to refer to all digitally delivered psychotherapeutic interventions, including apps and programmes for use on a computer, tablet, or smartphone. We do not use these terms to refer to standard face-to-face psychotherapy that takes place via video conferencing tools. e-mental health interventions can be delivered with various degrees of professional support, i.e. unguided/self-guided and guided [2]. Guidance is beneficial for the outcome of internet-delivered interventions [3] because guided treatment provides better outcomes than unguided treatment [2-4]

Blended treatment integrates face-to-face sessions and online therapy into one treatment protocol. Compared with stand-alone face-to-face psychotherapy, blended treatment can have several advantages. For example, adding online modules may enhance patient self-management. Additionally, the therapy structure may become more transparent and face-to-face sessions may be optimally used because they are prepared in the online environment [5]. Thus, blended treatment may save clinicians time. There is also evidence that blended treatment may lead to lower dropout rates and greater abstinence rates in patients with substance use or may help maintain in the long run any changes that were initially achieved during psychotherapy [6]. Psychotherapy in inpatient care was found to be more efficient after adding an online self-help programme to the treatment protocol [7]. Conceivably, blended treatment could be facilitated during inpatient care and may lead to the listed benefits. However, to date most studies investigating blended treatment protocols have focussed on outpatient care only $[8,9]$.

\section{Professionals' attitudes towards e-mental health}

Health care providers, such as doctors, psychologists and nursing staff, are the gatekeepers of health care delivery and the integration of digital health technologies into routine clinical practice [10]. In addition to legal and technical preconditions, acceptance of e-mental health by key stakeholders is relevant for the uptake of e-mental health into routine care [11].

The acceptance of e-mental health depends on certain predictors. Venkatesh et al. [12] formulated the Unified Theory of Acceptance and Use of Technology (UTAUT), which proposes four direct determinants of user acceptance and behaviour, i.e. performance expectancy, effort expectancy, social influence and facilitating conditions.
The first three are strong indicators of the intention to use; whereas the fourth, together with intention to use, predicts usage behaviour directly.

European mental health care professionals appear to be rather open towards therapeutic app use, with or without the support of a health care professional [13]. However, in many European countries, the acceptability of blended treatment is higher than that of unguided stand-alone internet treatments [14].

In contrast to the amount of research in the outpatient sector [15], little research has been performed on professionals' acceptance of e-mental health in German inpatient care. Hennemann et al. [16] surveyed 152 participants from four German rehabilitation hospitals with different diagnostic specialisations. In this multi-professional sample, doctors', psychologists', physical therapists', nurses', nutritionists' and others' intention to use e-health interventions during inpatient rehabilitation was rather low (5-point Likert scale: $M=2.47, \mathrm{SD}=0.9$ ); whereas, their acceptance of online aftercare was moderate $[M=3.08, \mathrm{SD}=0.96, t(127)=8.22$, $p<0.001]$. However, this study did not specifically examine the attitude towards e-mental health within psychiatric inpatient care. A first insight into stakeholders' attitude towards e-mental health in a German inpatient psychiatric hospital was given by Dorow et al. [17]. The group asked patients $(n=181)$ and mental health professionals $(n=31$, primarily psychologists and nurses) what they thought about implementing an internet-based self-management tool for treating depression in inpatient care. Most of the health care professionals stated that the presented self-management tool could be easily integrated into the hospital workflow as an additional therapy option and that they would recommend it, and patients also reported moderate to high user acceptance. Thus, the professionals tended to have a rather positive attitude. However, the sample was comparatively small [17]. Therefore, the question of how professionals think about e-mental health in psychiatric inpatient care remains open.

\section{Benefits of e-mental health}

The primary incentives to implement e-mental health into health care services, perceived by different organisations throughout Europe, are expected cost-effectiveness and increased access [14]. A recent systematic review revealed flexibility with timing and location as the main benefits of implementing e-mental health [18]. However, reaching patients that you might not reach otherwise cannot be seen as a benefit of e-mental health in inpatient treatment settings, where patients are already receiving care. Thus, the question remains whether e-mental health has additional advantages in inpatient care.

One of the main expected advantages of blended treatment is that clinicians can concentrate on process-related 
treatment components and use internet-delivered modules to deliver practical therapy components, such as assignments, diaries and psychoeducation [5]. Thus, compared with standalone face-to-face therapy, blended treatment may save clinicians time [6], although there is also evidence to the contrary [19]. Blended treatment may improve therapy quality and outcome: Dutch therapists working with blended cognitive behaviour therapy reported that the pre-set structure made them and their patients more adherent to the treatment protocol [19]. As mentioned above, blended treatment may also help to reduce dropout rates and to maintain changes achieved during psychotherapy [6].

To date, no studies have investigated the specific benefits of internet-delivered interventions in psychiatric inpatient care from the perspective of mental health care professionals.

\section{Barriers to the integration of e-mental health}

Several barriers may explain the rather low degree of implementation of e-mental health in routine health care. In 2014, different European stakeholders considered the low feasibility of delivery within existing health care systems to be a primary barrier for integrating internet-delivered psychotherapy [14]. Other barriers include the potential clinical inferiority of e-mental health compared with treatment as usual, limited internet literacy and access and suspected reluctance of mental health professionals and patients [14, $20]$, as well as the confidentiality and security of patient data [18].

In Germany, the main barriers perceived by professionals are data safety issues, technical problems, a severe course of disease [17] and insufficient e-mental health education and infrastructure [16]. Five German psychotherapists who implemented e-mental health into their outpatient therapy routine reported 'limited customizability and autonomy of decisions concerning blending the therapy', 'disease-related contraindications', 'negative affect caused by burden through technical problems' and 'hampered establishment of therapeutic alliance by technical issues' to be the main barriers [11]. There is also evidence that blended treatment is not time efficient [19] or cost effective from a societal perspective [9] compared with stand-alone face-to-face therapy. Further barriers may exist that are specific for psychiatric inpatient care and have not yet been investigated.

\section{Facilitators for the integration of e-mental health}

Titov et al. [21] searched for common features of hospitals that have implemented internet-delivered cognitive behaviour therapy (ICBT) successfully into routine care. They compared five hospitals in Sweden, Denmark, Norway, Canada and Australia. Thus, all five hospitals were in high-income countries with publicly funded health care and high internet use, all of which are obvious facilitators for e-mental health. The authors summarized eight key success factors, including 'strong clinical, Information Technology (IT) and organisational governance', 'use of well-developed and validated ICBT programmes', 'safe and effective provision of online therapy programmes' and 'well trained and supported therapists'. Recent systematic literature reviews revealed the acceptance of patients and professionals and the appropriateness of e-mental health in addressing patients' mental health disorders as facilitators [18, 22]. Davies et al. [18] found that health professionals supported online therapies only for patients with relatively straightforward, low-risk diagnoses, strong motivation and engagement, high computer literacy and access and low need for tailored content. The availability and reliability of the technology required for e-mental health applications and the interoperability of these applications with other existing technologies are further facilitators [22]. Additionally, researchers have emphasised the need for targeted training of professionals and organisational support to manage changed workflows [18]. Therapists who gained first experience with blended treatment reported that having sufficient training on how to work in a blended way and how to communicate online are key components for enabling online therapies [19]. However, research to date has not yet explicitly determined facilitators for e-mental health in inpatient psychiatric care in Germany from the professionals' point of view.

\section{The current study}

This study was part of the European project eMEN, which aims to increase knowledge on e-mental health implementation and was funded by the European Commission (Interreg North-West Europe Programme) [23, 24]. The study had two objectives. First, it aimed to assess the attitude of mental health care professionals working in inpatient care settings towards online therapy, regardless of the level of guidance. Second, it aimed to identify potential barriers, facilitators and benefits of the implementation of e-mental health in German psychiatric inpatient care from the professionals' perspective. We chose a mixed-methods approach to answer these research questions [25].

\section{Methods}

\section{Participants and procedure}

To ensure that not only those professionals with an affinity for the topic participated in our survey on e-mental health, 
rather than conducting an online survey, we engaged with professionals by holding workshops directly on site. We held the workshops (duration: $90 \mathrm{~min}$ ) at ten different psychiatric hospitals throughout Germany that were located in both urban and rural areas in the states of Schleswig-Holstein, Berlin, Brandenburg, North Rhine-Westphalia and Bavaria. The hospitals varied in size and in their approach to care, i.e. they consisted of both university and specialist hospitals, so that the study covered a wide range of settings. Existing weekly dates for internal training that are part of the hospital routine were used for the workshops. Thus, all health care professionals who treat patients with mental disorders were welcome to attend.

Two weeks in advance of the workshops, participants were provided with free access to the online therapy platform moodbuster (https://www.moodbuster.science/), which was developed by Vrije Universiteit Amsterdam, and asked to explore the online therapy modules on their own. We used moodbuster as an example to ensure that every participant had experienced an internet-delivered intervention before participating in the workshop. At the time of the workshops, moodbuster consisted of a cognitive behavioural therapy approach for depression. It offered six online modules that included psychoeducation, cognitive restructuring and behavioural activation. In moodbuster, texts and videos guide the user through the modules, which also use exercises and homework assignments. The moodbuster platform consists of a web portal for patients and practitioners and a mobile application with which many variables, such as mood and daily activities, can be monitored (also called ecological momentary assessment) [26].

In case any participants did not have the time or opportunity to learn about moodbuster or any other internetdelivered intervention in advance of the workshop, an expert of the moodbuster platform development team presented the programme at each on-site workshop (20min presentation). After the presentation, participants answered a paper-and-pencil survey consisting of closed questions on their attitudes towards internet-delivered interventions and open-response questions on perceived barriers, anticipated benefits and facilitators of internetdelivered intervention in inpatient mental health routine care (see measures section below for details). To further develop the moodbuster platform and to adapt it to inpatient care needs, participants were asked to fill in a usability questionnaire on moodbuster. The results of this questionnaire are not reported here, but please see the questionnaire in Supplementary Appendix A for details on usability items. After data collection, there was some time for discussion within the groups. However, that content was not coded and, thus, not analyzed and is not reported here.

\section{Measures}

This study took a mixed-methods approach [25]. Attitude towards e-mental health was assessed by items on an ordinal scale and scores were quantitatively analyzed. In contrast, the potential benefits, barriers and facilitators of implementing online therapy in inpatient routine care were assessed by open-response questions and answers were qualitatively analyzed with the inductive approach. Both methods and the data analyses are described in more detail below.

\section{Sociodemographic characteristics}

The survey consisted of items on age, sex, occupation, years of professional experience and time spent with moodbuster in preparation for the workshop. To maintain participants' anonymity, we did not record the psychiatric unit or the participants' exact workplace.

\section{Attitudes towards online therapy}

First, we evaluated the attitudes of mental health professionals towards e-mental health by the 8-item ATiPP questionnaire [27]. A 5-point Likert scale was used with anchors ranging from (1) 'I strongly disagree' to (5) 'I strongly agree'. For every participant, we calculated an ATiPP score by summing the item values and dividing this sum by the number of items answered. Thus, an individual's ATiPP score could range from 1 to 5 , with higher values indicating a more positive attitude towards online therapy. The ATiPP questionnaire was originally designed to assess attitudes towards telemedicine in psychiatry and psychotherapy and showed very good reliability (Cronbach's $\alpha>0.8$ ). For the current study, the broader concept of telemedical interventions was narrowed down to the use of online therapy. The adapted questionnaire showed an average inter-item covariance of 0.22 and good reliability (Cronbach's $\alpha=0.73$ ). For the items, see Supplementary Appendix A, questions 1-8. See Supplementary Appendix $\mathrm{B}$ for details on the psychometrics of the adapted ATiPP questionnaire.

Second, we evaluated the intention to use online therapy in inpatient care as a proxy for attitude (see Supplementary Appendix A, question 23).

Third, we assessed social influence, i.e. the expected attitude of others, on a 5-point scale (see Supplementary Appendix A, questions 24-25). Social influence is a predictor of behavioural intention to use the technology [12]. 


\section{Potential benefits, barriers and facilitators of implementing online therapy in inpatient routine care}

The survey of benefits, barriers and facilitators of implementing online therapy in inpatient routine care was inspired by the UTAUT model predictors [12]. Questions from previous research were adapted to meet the needs of the current study. Personal IT literacy and experience with online therapy programmes were assessed (see Supplementary Appendix A, questions 21-22). Additionally, we evaluated potential barriers and facilitators, i.e. preconditions, that need to be fulfilled and the perceived usefulness and potential benefits of online therapy in inpatient care. An openresponse format was used for the respective questions (see Supplementary Appendix A, questions 26-36).

\section{Data analysis}

In addition to quantitative descriptive analysis of the scale scores [mean values $(M)$, standard deviations (SD), frequencies, and percentages], we performed a qualitative analysis of the responses to the open questions, as follows: in accordance with the inductive approach [28], codes were developed by paraphrasing and summarising the raw data that addressed potential benefits, barriers and facilitators of online therapy in psychiatric inpatient care. Raw data from free text responses were coded by one psychologist who was involved in data collection during the workshops. By paraphrasing and summarizing the responses, she first developed a list of codes that were based on $40 \%$ of material. Then, she searched the rest of the qualitative data material (60\%) for statements related to the original code list. During this coding process, she added new codes and adjusted the existing ones to fit the data. A sentence or paragraph could be assigned to one or more codes. The number of participants that referred to a code was counted. All qualitative analyses were conducted with the software MaxQDA Analytics Pro 2020 (Release 20.0.07). The raw data with assigned codes are available from the corresponding author.

\section{Results}

In total, 176 participants aged between 20 and 65 years $(M=37.6, \mathrm{SD}=11.5)$ answered the questionnaire; $67 \%$ of participants were females. Occupation was specified by 171 participants, as follows: 81 stated that they had a background in psychology, 74 were medical doctors (36 of whom were doctors in training) and 16 described themselves as nursing or other health care staff in psychiatry. A great majority of participants described their personal experience with online therapy as poor (Table 1).
Table 1 How much experience have you gained with online therapies?

\begin{tabular}{llll}
\hline & $n$ & $\%$ & Cumulative \% \\
\hline [0] Very little & 136 & 77 & 77 \\
1 & 27 & 15 & 93 \\
2 & 9 & 5 & 98 \\
3 & 3 & 2 & 99 \\
[4] A lot & 1 & 1 & 100 \\
Total & 176 & 100 & 100 \\
\hline
\end{tabular}

Table 2 How common is knowledge about internet-based interventions for the treatment of mental illness in the hospital where you work?

\begin{tabular}{llll}
\hline & $n$ & $\%$ & Cumulative \% \\
\hline [1] A little & 65 & 38 & 38 \\
2 & 71 & 41 & 79 \\
3 & 30 & 17 & 97 \\
4 & 5 & 3 & 99 \\
[5] Very & 1 & 1 & 100 \\
Total & 172 & 100 & \\
\hline
\end{tabular}

Also, the knowledge about online therapy for the treatment of mental illness in one's own hospital was rated as being rather low (Table 2).

\section{Attitudes towards online therapy in inpatient care}

The first objective of the study was to assess the attitude of German mental health care professionals working in an inpatient care setting towards online therapy. The participants' answers tended towards the center of the 8-item ATiPP scale $\left(N=172\right.$, Median $_{\text {ATiPP score }}=3.6, M_{\text {ATiPP score }}=3.6$, $\mathrm{SD}=0.54, \min =2, \max =4.8)$. We found no significant association between the attitude towards online therapy (ATiPP score) and occupation, age, professional experience or personal experience with online therapy. However, we did find a small, significant, positive correlation of the ATiPP score with self-evaluated IT knowledge and skills $(r=0.15$, $p<0.05)$. Results on the item level showed that most participants agreed or strongly agreed that 'generally, online therapy is a good addition to medical services' (77\%). However, only $30 \%$ agreed that 'an effective treatment of patients with mental illness is possible via online therapy'. And only $36 \%$ 'would recommend that their patients receiving psychiatric or psychotherapeutic treatment need an online therapy if such were to be offered'. For full ATiPP results on the item level, please see Supplementary Appendix B.

About $21 \%$ of the participants had rather little intention to use online therapy in their daily routine and almost 
Table 3 How much do you like the idea of using online therapy in inpatient routine care?

\begin{tabular}{llll}
\hline & $n$ & $\%$ & Cumulative \% \\
\hline [1] A little & 11 & 6 & 6 \\
2 & 25 & 14 & 21 \\
3 & 55 & 32 & 52 \\
4 & 57 & 33 & 85 \\
[5] A lot & 26 & 15 & 100 \\
Total & 174 & 100 & \\
\hline
\end{tabular}

a third was indifferent. However, about half (48\%) were interested in implementing online therapy in their daily routines in inpatient care (Table 3 ).

In comparison with their own attitude, participants considered the attitude of others (family, friends and acquaintances) towards online therapy to be more positive. Three-quarters of those surveyed believed that their family, friends and acquaintances have a positive (37\%) or very positive (39\%) attitude towards online therapies to treat mental illness (Table 4).

In contrast, many participants suspected that their colleagues have a rather negative or indifferent attitude towards online therapy and only $22 \%$ thought that their colleagues have a positive $(20 \%)$ or very positive $(2 \%)$ attitude towards implementing online therapies in their daily work (Table 5). Thus, these results suggested that work peer influence has a rather negative effect on the behavioural intention to use online therapies. In contrast, the social influence of family, friends and acquaintances was likely to be more positive. The individual ATTiP score was positively correlated with the assumed attitude of family, friends and acquaintances $(r=0.31, p<0.001)$ and colleagues $(r=0.20, p<0.05)$ towards online therapy.

Table 4 What attitude do you assume your family and friends/ acquaintances have towards the use of online therapies for treatment of mental illness?

\begin{tabular}{llll}
\hline & $n$ & $\%$ & Cumulative \% \\
\hline [1] Very negative & 6 & 3 & 3 \\
2 & 28 & 16 & 19 \\
3 & 1 & 1 & 20 \\
4 & 65 & 37 & 57 \\
{$[5]$ Very positive } & 67 & 39 & 100 \\
Total & 174 & 100 & \\
\hline
\end{tabular}

Table 5 What attitude do you assume your colleagues have towards the use of online therapies in inpatient routine care?

\begin{tabular}{llll}
\hline & $n$ & $\%$ & Cumulative \% \\
\hline [1] Very negative & 3 & 2 & 2 \\
2 & 47 & 27 & 29 \\
3 & 86 & 49 & 78 \\
4 & 35 & 20 & 98 \\
[5] Very positive & 3 & 2 & 100 \\
Total & 174 & 100 & \\
\hline
\end{tabular}

\section{Potential benefits, barriers and facilitators of the implementation of online therapy in inpatient care}

\section{Benefits of e-mental health in inpatient care}

Mental health professionals identified 18 potential benefits of online therapy in psychiatric inpatient care. Optimised treatment structure through online therapy was listed as an important potential benefit by $32 \%(n=57)$ of participants. Furthermore, $31 \%(n=55)$ classified online therapy programmes as a helpful add-on to inpatient face-to-face therapy, in terms of an extension of the treatment spectrum. In particular the empowerment of the patient (26\%; $n=45$ ) (e.g. through the possibility to work independently from the therapist) and the meaningful use of treatment-free times during hospitalisation $(22 \% ; n=39)$ were mentioned as potential benefits. Psychoeducation through online programmes, which present the information well in writing, by videos, etc., and make it accessible at any time, was also considered useful $(26 \% ; n=45)$. In addition, many of the professionals anticipated a higher level of treatment satisfaction and better adherence of patients as a result of the additional treatment option $(26 \% ; n=45)$. Furthermore, better documentation, symptom tracking, monitoring and evaluation of therapy progress and its transparency to the patient could be positive results of implementing online therapy in inpatient care $(23 \% ; n=41)$. Also, relapse prevention and bridging the transition from inpatient to outpatient care were stated as potential benefits $(19 \% ; n=34) ; 14$ participants (8\%) mentioned that only outpatient care would benefit from online therapy. For full results on potential benefits, please see the coded responses and their frequencies listed in Table 6.

\section{Barriers to the integration of e-mental health in inpatient care}

Mental health professionals identified 20 barriers to implementation of online therapy in psychiatric inpatient care. The barrier most frequently anticipated by health care 
Table 6 Potential benefits of implementing online therapy in psychiatric inpatient care- the view of health care professionals

\begin{tabular}{lll}
\hline Codes & Participants $N$ & $\%$ \\
\hline Optimised treatment structure & 57 & 32 \\
Add-on to face-to-face therapy & 55 & 31 \\
Empowerment of patients & 45 & 26 \\
Helpful for psychoeducation & 45 & 26 \\
Better adherence and satisfaction/more interesting for patients & 45 & 26 \\
Better documentation/monitoring/therapy evaluation & 41 & 23 \\
Using treatment-free times in hospital meaningfully & 39 & 22 \\
Intensifying therapy success & 34 & 19 \\
Relapse prevention/bridging from in- to outpatient care & 34 & 19 \\
Time saving/reduced workload & 28 & 16 \\
Standardised interventions & 14 & 8 \\
(Benefits for outpatient care only) & 14 & 8 \\
Bridging waiting time & 14 & 8 \\
High scalability/individualization & 11 & 6 \\
Facilitating access to psychotherapy & 10 & 6 \\
Economization/fewer staff necessary & 8 & 5 \\
Larger number of patients can be treated & 8 & 5 \\
Multilingual treatment opportunities & 6 & 3 \\
Crisis planning & 1 & 14 \\
Participants WITH statements on benefits & 152 & 24 \\
Participants WITHOUT statements on benefits & 176 & 100 \\
Total number of participants & & 56
\end{tabular}

professionals in psychiatric hospitals was that patients may lack the necessary capabilities, mainly because their symptoms are too severe and they would be cognitively incapable (23\%; $n=41)$. Another common argument against online therapy was that the important face-to-face contact would be neglected $(22 \% ; n=39)$. Further barriers mentioned were a shortage of technical equipment $(21 \% ; n=37)$ and lack of internet access $(13 \% ; n=23)$. Additionally, mental health care professionals assumed that patients might not be willing to do online therapy during inpatient care $(19 \% ; n=33)$. Also mentioned was that a lack of acceptance by staff might be a significant barrier, i.e. colleagues may not be convinced and fear being replaced by the technology $(14 \% ; n=25)$. All coded responses that addressed barriers are listed in Table 7.

\section{Facilitators to the integration of e-mental health in inpatient care}

In total, mental health professionals identified 34 different potential facilitators for implementing online therapy in psychiatric inpatient care. Regarding technical preconditions for successful implementation into inpatient care, very good usability of the online therapy programme $(32 \% ; n=57)$, free internet access $(20 \% ; n=35)$ and devices $(22 \% ; n=39)$ for patients and health care professionals were of particular importance. Furthermore, professionals considered it very important that the online therapy is highly adaptive to different needs and patients $(20 \% ; n=35)$, that data security is ensured $(19 \% ; n=34)$ and that the system interoperates with the local hospital IT system to avoid increased amounts of documentation $(10 \% ; n=18)$.

Regarding patients, participants saw sufficient cognitive ability and functional level as indispensable preconditions for applying online therapy in inpatient care $(38 \% ; n=68)$. Patients in severe crisis who are being treated on acute care units may not be able to concentrate on online therapy. Additionally, patients should participate voluntarily and be intrinsically motivated to work online $(14 \% ; n=25)$.

The most important facilitator on the organisational side was the further education of staff. Many participants identified training as the necessary next step towards implementation of online therapy $(33 \% ; n=58)$. Clearly structured workflows for the application and integration of online therapy in everyday clinical practice were also rated as particularly important $(14 \% ; n=25)$. By this, the professionals meant, for example, that online therapy should be a useful addition to existing therapies and not have a different content. Acceptance by mental health care professionals (14\%; $n=25)$ and transparent communication to patients about the treatment option were also key $(14 \% ; n=25)$. Some professionals mentioned that face-to-face therapy should not be replaced by online therapy but rather remain an additional option $(11 \% ; n=20)$. The coded responses and the number of participants that gave these responses are listed in Table 8 . 
Table 7 Potential barriers to implementing online therapy in psychiatric inpatient care-the view of health care professionals

\begin{tabular}{lll}
\hline Codes & Participants $N$ & $\%$ \\
\hline Patients not capable & 41 & 23 \\
Neglect of face-to-face contacts & 39 & 22 \\
No workspaces, technical equipment & 37 & 21 \\
Patients not willing & 33 & 19 \\
No resources (financial, personal) & 30 & 17 \\
Staff not convinced, fear being replaced & 25 & 14 \\
No (free wireless) internet access in hospital & 23 & 13 \\
Impersonal treatment, depersonalization of therapy & 17 & 10 \\
High workload for professionals & 16 & 9 \\
Not efficient (yet) & 16 & 9 \\
Data not secure & 13 & 7 \\
Lack of knowledge among staff & 11 & 6 \\
Encourages withdrawal of patients into the digital world & 10 & 6 \\
No benefits for inpatient care & 7 & 4 \\
Programmes not sufficiently user-friendly & 5 & 3 \\
No time & 1 & 1 \\
Lack of control by the practitioner & 1 & 1 \\
Suitable patients difficult to identify & 1 & 1 \\
Job cuts for psychologists & 1 & 1 \\
Psychodynamic therapy approaches unclear & 1 & 1 \\
Number of participants WITH statements on barriers & 139 & 79 \\
Number of participants WITHOUT statements on barriers & 37 & 21 \\
Number of participants & 176 & 100 \\
\hline
\end{tabular}

\section{Discussion}

We found that many mental health care professionals were not sure of how to evaluate online therapy in a psychiatric inpatient care setting. This may be related to the reported lack of practical experience. Nevertheless, $48 \%$ of the participants were interested in using online therapy in their daily routine. Thus, health care professionals in psychiatry seem to have a slightly more positive attitude towards e-health than their colleagues in inpatient rehabilitation centres with different diagnostic orientations, only $12 \%$ of whom showed a high level of intention to use e-health [28].

In line with an earlier study in which therapists who performed blended cognitive behavioural therapy were interviewed [19], the current study found that the most frequently mentioned potential benefit of the implementation of e-mental health in German inpatient care was providing better structured psychotherapeutic treatment. Further benefits were being an attractive add-on to face-to-face treatment and empowering patients. Nevertheless, our results on benefits suggest that for professionals a blended format that replaces parts of the current face-to-face interactions by digital tools would be more difficult to accept than e-mental health as add-on. To our knowledge, to date, no other study has assessed the potential benefits of online interventions in
German psychiatric inpatient routine care from the view of the mental health professionals.

Regarding perceived barriers to implementation of online therapy in routine psychiatric inpatient care in Germany, this study reveals the serious concern that patients admitted to hospital because of the severity of their illness may not be willing or cognitively and mentally able to partake in online therapy. Additionally, mental health professionals worry that important face-to-face contacts would be neglected. Furthermore, they point to a lack of financial and personnel resources. In accordance with the present results, previous studies demonstrated that a severe course of disease $[11,17]$ and insufficient (technical) infrastructure [16] are perceived obstacles that prevent mental health professionals from using digital interventions in Germany and other Western societies [18]. The lack of infrastructure is particularly relevant in deprived and homeless patients with a high mental health burden because they will not be able to continue digital interventions after discharge [29].

Concerning facilitators, this study identified several factors that would be necessary for the implementation of e-mental health in the view of mental health care professionals working in psychiatric inpatient care in Germany. Amongst others, these facilitators include technical preconditions (internet access, workspaces, devices), usability of internet-delivered interventions, sufficient functional level of 
Table 8 Facilitators to implement online therapy in psychiatric inpatient care-the view of health care professionals

\begin{tabular}{|c|c|c|c|}
\hline Category & Codes & Participants $N$ & $\%$ \\
\hline \multirow[t]{10}{*}{ Technical facilitators } & Easy handling/high accessibility/usability & 57 & 32 \\
\hline & Workspaces with computer/tablets/smartphones & 39 & 22 \\
\hline & Free wireless internet access for patients & 35 & 20 \\
\hline & High adaptability/individualization of programme & 35 & 20 \\
\hline & Data security & 34 & 19 \\
\hline & Overall technical preconditions & 34 & 19 \\
\hline & Interoperability of programme & 18 & 10 \\
\hline & Stability of programme & 12 & 7 \\
\hline & Technical support available & 4 & 2 \\
\hline & Offline mode available & 2 & 1 \\
\hline \multirow[t]{8}{*}{ Facilitating patient characteristics } & Sufficient functional level of the patient & 68 & 39 \\
\hline & Compliant/motivated patients & 25 & 14 \\
\hline & Young patients & 18 & 10 \\
\hline & Acceptance by patients & 15 & 9 \\
\hline & Patients with affinity for technology & 14 & 8 \\
\hline & First admission to hospital & 3 & 2 \\
\hline & Experienced in psychotherapy & 2 & 1 \\
\hline & No problems of loneliness & 1 & 1 \\
\hline \multirow[t]{16}{*}{ Organisational facilitators } & Further education/training of staff & 58 & 33 \\
\hline & Clear workflow structure & 25 & 14 \\
\hline & Informing patients & 25 & 14 \\
\hline & Acceptance by staff & 25 & 14 \\
\hline & No substitution of face-to-face contact & 20 & 11 \\
\hline & Financing/availability of logins & 19 & 11 \\
\hline & Relating therapist must stay present & 18 & 10 \\
\hline & More experience & 7 & 4 \\
\hline & More research & 7 & 4 \\
\hline & (Time) efficiency & 6 & 3 \\
\hline & Personnel resources & 6 & 3 \\
\hline & More evidence & 5 & 3 \\
\hline & Trust & 4 & 2 \\
\hline & Openness of the hospital management & 4 & 2 \\
\hline & Defined inclusion and exclusion & 2 & 1 \\
\hline & Legal basis for the prevention of risks & 1 & 1 \\
\hline No barriers to overcome-no actions are required & & 10 & 6 \\
\hline Number of participants WITH statements on facilitators & & 156 & 89 \\
\hline Number of participants WITHOUT statements on facilitators & & 20 & 11 \\
\hline Number of participants & & 176 & 100 \\
\hline
\end{tabular}

patients, and further training of staff. In particular the technical aspects (availability, reliability and interoperability) and the appropriateness of the internet-delivered interventions for addressing a patient's mental health problem are also reflected in an earlier systematic review of implementation research [22]. The need for targeted training and support of professionals with clear transparent protocols was also emphasised by a recent review by Davies et al. on health care professionals' perceptions of web-based psychological treatments in routine care [18]. Probably because most of the patients in psychiatric inpatient care are severely impaired, the professionals in the present study placed greater emphasis on the fact that a certain level of functioning in patients is a prerequisite for the use of e-mental health. The other reviews mentioned above included all possible treatment settings but more frequently examined scenarios in the outpatient setting.

Taken together, these findings about barriers and facilitators suggest that a successful implementation of online therapy in psychiatric inpatient routine care would work only 
if technical preconditions were met and if the internet-delivered interventions were tailored to the needs of psychiatric hospitals, i.e. high usability for patients and professionals. It is especially important to involve mental health professionals in the selection of online therapy programmes because individual needs vary considerably. However, studies on telepsychiatry, i.e. treatment via videoconferencing, suggest that our findings may not be specific to internet-delivered interventions [30-32]. The concerns of mental health care professionals regarding therapeutic alliance, privacy, safety and technology issues and their demands regarding financing, credentialing and education are very similar and universally valid [18]. Thus, the extensive adaptation of the clinical workflow is essential to the mental health care professional's acceptance of e-health options, irrespective of the technology.

\section{Limitations}

The participants in this study lacked practical experience with e-mental health so the reported benefits, barriers and facilitators of online therapy in psychiatric inpatient care remain rather hypothetical. Despite the 2-week test period with the moodbuster platform to prepare for the workshops, $77 \%$ of participants indicated having no experience at all with online interventions. Other studies revealed that professionals' attitude towards online therapy improves with more frequent use [19]. Implementation studies should be undertaken to investigate benefits, barriers and facilitators and also to compare online therapy as an add-on with blended therapy in psychiatric inpatient care in practice.

A methodological shortcoming of the study is that responses to the open-format questions were coded by only one coder. However, the questions regarding potential benefits, barriers and facilitators of e-mental health in psychiatric inpatient care were very precise and the short written answers left little room for interpretation. Therefore, we assume that this shortcoming did not cause any bias in the results.

Moreover, it is important to bear in mind that the present data were collected before the 2020 global corona pandemic, which represented a high mental health burden and required digital interventions to avoid infections [33, 34]. Today, many areas of life have been switched to digital solutions without personal contact. This includes psychiatric care, which partially introduced telepsychiatry, i.e. treatment via telephone or video [35]. In Germany, many doctors currently expect that even after the pandemic, video consultation will be used more often than it was before [36]. We assume that mental health care professionals' attitude towards online treatment as an addon or in a blended format will also further improve as a result of the recent experiences. To gain further information on this topic, we recommend that future studies evaluate professionals' view of online therapy and implementations during and after the global corona pandemic.

\section{Conclusion}

This study sheds light on the specific benefits, barriers and facilitators concerning the implementation of e-mental health in psychiatric inpatient care. It shows that mental health care professionals in Germany see potential added value of internetdelivered interventions for inpatient routine care, for instance regarding better structured psychotherapy and patient empowerment. These benefits seem to make it worthwhile to tackle the barriers to implementation. Besides technical preconditions on the organisational level and further education of staff, the features of the internet-delivered interventions appear to be crucial facilitators.

To be accepted, internet-delivered interventions should be highly interoperable with a hospitals' existing digital documentation system and capable of being integrated into the inpatient workflow. In addition, online therapy should be easy to use for patients and professionals. Especially for inpatient care, the cognitive demands that the online therapy places on the patient should be highly adjustable by professionals. In line with the proposed actions by the European transnational policy for e-mental health [37], we recommend strengthening implementation research on the organisational level.

Supplementary Information The online version contains supplementary material available at https://doi.org/10.1007/s00406-021-01251-1.

Acknowledgements We would like to thank the participants and the management of the participating hospitals for supporting this survey.

Funding The eMEN project received funding from the European Union's Interreg Northwest Europe programme.

Availability of data and material The raw data with assigned codes in MaxQDA Software are available from the first author, Julia Sander: j.sander@dgppn.de.

Code availability Not applicable.

\section{Declarations}

Conflict of interest The authors declare that they have no conflicts of interest.

\section{References}

1. Andersson G, Carlbring P, Titov N, Lindefors N (2019) Internet interventions for adults with anxiety and mood disorders: a narrative umbrella review of recent meta-analyses. Can J Psychiatry 64(7):465-470. https://doi.org/10.1177/0706743719839381 
2. Johansson R, Andersson G (2012) Internet-based psychological treatments for depression. Expert Rev Neurother 12(7):861-870. https://doi.org/10.1586/ern.12.63

3. Baumeister H, Reichler L, Munzinger M et al (2014) The impact of guidance on internet-based mental health interventions-a systematic review. Internet Interv 1:205-215. https://doi.org/10. 1016/j.invent.2014.08.003

4. Carlbring P, Andersson G, Cuijpers P, Riper H, Hedman-Lagerlöf E (2018) Internet-based vs. face-to-face cognitive behavior therapy for psychiatric and somatic disorders: an updated systematic review and meta-analysis. Cogn Behav Ther 47(1):1-18. https:// doi.org/10.1080/16506073.2017.1401115

5. van der Vaart R, Witting M, Riper H et al (2014) Blending online therapy into regular face-to-face therapy for depression: content, ratio and preconditions according to patients and therapists using a Delphi study. BMC Psychiatry 14:355. https://doi.org/10.1186/ s12888-014-0355-Z

6. Erbe D, Eichert HC, Riper H, Ebert DD (2017) Blending faceto-face and internet-based interventions for the treatment of mental disorders in adults: systematic review. J Med Internet Res 19(9):e306. https://doi.org/10.2196/jmir.6588

7. Zwerenz R, Becker J, Knickenberg RJ, Siepmann M, Hagen K, Beutel ME (2017) Online self-help as an add-on to inpatient psychotherapy: efficacy of a new blended treatment approach. Psychother Psychosom 86(6):341-350. https://doi.org/10.1159/00048 1177

8. Kenter RMF, van de Ven PM, Cuijpers P et al (2015) Costs and effects of internet cognitive behavioral treatment blended with face-to-face treatment: results from a naturalistic study. Internet Interv 2(1):77-83. https://doi.org/10.1016/j.invent.2015.01.001

9. Kooistra LC, Wiersma JE, Ruwaard J et al (2019) Cost and effectiveness of blended versus standard cognitive behavioral therapy for outpatients with depression in routine specialized mental health care: pilot randomized controlled trial. J Med Internet Res 21(10):e14261. https://www.jmir.org/2019/10/e14261/. Accessed 11 Mar 2021

10. Leigh S, Ashall-Payne L (2019) The role of health-care providers in mHealth adoption. Lancet Digital Health 1(2):e58-e59. https:// doi.org/10.1016/S2589-7500(19)30025-1

11. Titzler I, Saruhanjan K, Berking M, Riper H, Ebert DD (2018) Barriers and facilitators for the implementation of blended psychotherapy for depression: a qualitative pilot study of therapists perspective. Internet Interv 12:150-164. https://doi.org/10.1016/j. invent.2018.01.002

12. Venkatesh V, Morris M, Davis G, Davis F (2003) User acceptance of information technology: toward a unified view. MIS Q 27(3):425-478. http://www.jstor.org/stable/30036540. Accessed 11 Mar 2021

13. Kerst A, Zielasek J, Gaebel W (2019) Smartphone applications for depression: a systematic literature review and a survey of health care professionals' attitudes towards their use in clinical practice. Eur Arch Psychiatry Clin Neurosci 270:139-152. https://doi.org/ 10.1007/s00406-018-0974-3

14. Topooco N, Riper H, Araya R et al (2017) Attitudes towards digital treatment for depression: a European stakeholder survey. Internet Interv 8:1-9. https://doi.org/10.1016/j.invent.2017.01.001

15. Dockweiler C, Kupitz A, Palmdorf S et al (2020) Onlinetherapie bei depressiven Störungen [Online therapy for depressive disorders]. Nervenarzt 91:243-251. https://doi.org/10.1007/ s00115-019-0730-6

16. Hennemann S, Beutel M, Zwerenz R (2017) Ready for eHealth? Health professionals' acceptance and adoption of ehealth interventions in inpatient routine care. J Health Commun 22(3):274-284. https://doi.org/10.1080/10810730.2017.1284286

17. Dorow M, Stein J, Förster F et al (2018) Implementation of the internet-based self-management program "moodgym" in patients with depressive disorders in inpatient clinical settingspatient and expert perspectives. Psychiatr Prax 45(5):256-262. https://doi.org/10.1055/s-0043-117049

18. Davies F, Shepherd HL, Beatty L, Clark B, Butow P, Shaw J (2020) Implementing web-based therapy in routine mental health care: systematic review of health professionals' perspectives. J Med Internet Res 22(7):e17362. https://doi.org/10.2196/ 17362

19. Mol M, van Genugten C, Dozeman E et al (2019) Why uptake of blended internet-based interventions for depression is challenging: a qualitative study on therapists' perspectives. J Clin Med 9(1):91. https://doi.org/10.3390/jcm9010091

20. Breedvelt JJ, Zamperoni V, Kessler D et al (2019) GPs' attitudes towards digital technologies for depression: an online survey in primary care. Br J Gen Pract 69(680):e164-e170. https://doi.org/ 10.3399/bjgp18X7007

21. Titov N, Dear B, Nielssen O et al (2018) ICBT in routine care: a descriptive analysis of successful clinics in five countries. Internet Interv 13:108-115. https://doi.org/10.1016/j.invent.2018.07.006

22. Vis C, Mol M, Kleiboer A et al (2018) Improving implementation of emental health for mood disorders in routine practice: systematic review of barriers and facilitating factors. JMIR Ment Health 5(1):e20. https://doi.org/10.2196/mental.9769

23. e-Mental Health Innovation and Transnational Implementation Platform North West Europe (eMEN) (2020) Project summary. https://www.nweurope.eu/projects/project-search/e-mental-healthinnovation-and-transnational-implementation-platform-northwest-europe-emen/undefined Accessed 17020

24. Gaebel W, Lukies R, Kerst A et al (2020) Upscaling e-mental health in Europe: a six-country qualitative analysis and policy recommendations from the eMEN project. Eur Arch Psychiatry Clin Neurosci. https://doi.org/10.1007/s00406-020-01133-y

25. Creswell J (2003) Research design: Qualitative, quantitative, and mixed methods approaches, 4 th edn. Sage, Thousand Oaks

26. Ebner-Priemer UW, Trull TJ (2009) Ecological momentary assessment of mood disorders and mood dysregulation. Psychol Assess 21(4):463-475. https://doi.org/10.1037/a0017075

27. Tonn P, Reuter SC, Kuchler I et al (2017) Development of a questionnaire to measure the attitudes of laypeople, physicians, and psychotherapists toward telemedicine in mental health. JMIR Ment Health 4(4):e39. https://doi.org/10.2196/mental.6802

28. Mayring P (2010) Qualitative Inhaltsanalyse: Grundlagen und Techniken [Qualitative content analysis: basics and techniques], 11 th edn. Beltz, Weinheim

29. Schreiter S, Bermpohl F, Krausz M et al (2017) The prevalence of mental illness in homeless people in Germany. Dtsch Arztebl Int 6114(40):665-672. https://doi.org/10.3238/arztebl.2017.0665

30. Cowan KE, McKean AJ, Gentry MT, Hilty DM (2019) Barriers to use of telepsychiatry: clinicians as gatekeepers. Mayo Clin Proc 94(12):2510-2523. https://doi.org/10.1016/j.mayocp.2019.04.018

31. Hubley S, Lynch SB, Schneck C, Thomas M, Shore J (2016) Review of key telepsychiatry outcomes. World J Psychiatry 6(2):269-282. https://doi.org/10.5498/wjp.v6.i2.269

32. Mitchell SA, MacLaren AT, Morton M, Carachi R (2009) Professional opinions of the use of telemedicine in child and adolescent psychiatry. Scott Med J 54:13-16. https://doi.org/10.1258/rsmsmj. 54.3 .13

33. Pierce M, Hope H, Ford T et al (2020) Mental health before and during the COVID-19 pandemic: a longitudinal probability sample survey of the UK population. Lancet Psychiatry 7(10):883892. https://doi.org/10.1016/S2215-0366(20)30308-4

34. Liu S, Heinz A (2020) Cross-cultural validity of psychological distress measurement during the coronavirus pandemic. Pharmacopsychiatry 53(5):237-238. https://doi.org/10.1055/a-1190-5029

35. Shore JH, Schneck CD, Mishkind MC (2020) Telepsychiatry and the coronavirus disease 2019 pandemic — current and future 
outcomes of the rapid virtualization of psychiatric care. JAMA Psychiatry. https://doi.org/10.1001/jamapsychiatry.2020.1643

36. Obermann K et al (2020) Ärztliche Arbeit und Nutzung von Videosprechstunden während der COVID-19-Pandemie. Eine gemeinsame repräsentative deutschlandweite Befragung ambulant tätiger Ärztinnen und Ärzte durch die Stiftung Gesundheit in Zusammenarbeit mit dem health innovation hub [Medical work and use of video consultations during the Covid 19 pandemic - a joint representative survey of physicians working in outpatient care throughout Germany by the Stiftung Gesundheit in cooperation with the health innovation hub] Ärzte im Zukunftsmarkt Gesundheit 2020. https://www.stiftung-gesundheit.de/pdf/studien/aerzteim-zukunftsmarkt-gesundheit_2020.pdf Accessed 17 Sept 2020

37. Gaebel W, Trost N, Diekmann S, Lukies R, Zielasek J (2019) Transnational policy for e-mental health: a guidance document for European policymakers and stakeholders. Co-funded by the EU Interreg North- West-Europe programme. LVR-Institute for Healthcare Research, Düsseldorf/Köln. https://www.nweurope.eu/ media/10450/emen_transnational-policy-for-e-mental-health_ guidance-document_3-2020.pdf Accessed 22 Sept 2020 